\title{
Symbolic computations of nonlinear observability
}

\author{
Ezequiel Bianco-Martinez, ${ }^{1}$ Murilo S. Baptista, ${ }^{1}$ and Christophe Letellier ${ }^{2}$ \\ ${ }^{1}$ Institute for Complex Systems and Mathematical Biology, SUPA, University of Aberdeen, Old Aberdeen AB24 3UE, United Kingdom \\ ${ }^{2}$ CORIA-UMR 6614 Normandie Université, CNRS-Université et INSA de Rouen, Campus Universitaire du Madrillet, \\ F-76800 Saint-Etienne du Rouvray, France
}

(Received 22 December 2014; published 18 June 2015)

\begin{abstract}
Observability is a very useful concept for determining whether the dynamics of complicated systems can be correctly reconstructed from a single (univariate or multivariate) time series. When the governing equations of dynamical systems are high-dimensional and/or rational, analytical computations of observability coefficients produce large polynomial functions with a number of terms that become exponentially large with the dimension and the nature of the system. In order to overcome this difficulty, we introduce here a symbolic observability coefficient based on a symbolic computation of the determinant of the observability matrix. The computation of such coefficients is straightforward and can be easily analytically carried out, as demonstrated in this paper for a five-dimensional rational system.
\end{abstract}

DOI: 10.1103/PhysRevE.91.062912

PACS number(s): 05.45.Ac, 05.45.Jn

\section{INTRODUCTION}

When an experimental system is investigated, it is not possible to measure all the variables required for describing its state fully. Consequently, a space is reconstructed from the variables measured. This can be achieved by reconstructing the nonmeasured variables from the measured ones by using delay or derivative coordinates [1,2], both sets being equivalent [3] for providing a way to fully reconstruct the space from well behaved variables. One of the natural questions which thus arise is to determine whether all measured variables can equivalently fully reconstruct the state space. A very useful concept for addressing such a question is the concept of observability for nonlinear systems as introduced by Hermann and Kerner [4]. It is relevant to point out that a nonlinear theory must be used in order to avoid too many oversimplifications, which can lead to erroneous results, particularly when rational systems (systems whose governing equations are rational functions) are considered. It was shown that the choice of the variable may deeply affect the quality of the reconstructed space $[5,6]$. In other words, there are some variables which provide a better observability of the original state space than others.

Unfortunately, and despite some attempts [7], it is still an open problem to address such a question when only experimental data are available. On the other hand, observability can be rigorously determined when the governing equations - the set of differential equations - are known. Moreover, the concept of observability is strongly related to the determinant of a so-called observability matrix (introduced in Sec. II) which can be already quite complicated to compute for systems whose dimension is not very large, particularly when these systems are rational.

Moreover, it was intuitively understood that between a full observability provided by a set of measurements and a total lack of observability (for which there is not even a single state which can be completely reconstructed from the measurements), there are some measurements making it possible to reconstruct only a subset of the original state space of the system. A numerical estimation of the observability was thus proposed by using the so-called observability coefficients $[5,6,8]$ when derivative coordinates are used. The case of delay coordinates was also recently addressed [9]. Unfortunately, these calculations of observability coefficients combine analytical computations with numerical simulation and are only feasible for paradigmatic, but quite simple systems.

To quantify observability of (i) polynomial and (ii) lowdimensional systems, a symbolic observability coefficient based on a graphical interpretation of the flow graph similar to the one developed in [10] was introduced in [11]. The aim of this paper is to extend that work and show how observability can be quantified for higher-dimensional and rational systems by a different definition of a symbolic observability coefficient. The subsequent part of this paper is organized as follows. In Sec. II, the relevant concepts for the observability of nonlinear systems are briefly introduced and the procedure to compute the new symbolic observability coefficient is described. In Sec. III, many examples are treated to validate this new symbolic observability coefficient including a highdimensional rational system. Sec. IV gives conclusions.

\section{SYMBOLIC OBSERVABILITY COEFFICIENTS}

\section{A. Differential embeddings and observability matrix}

Let us start with a nonlinear dynamical system,

$$
\dot{x}_{i}=f_{i}\left(x_{1}, x_{2}, x_{3}, \ldots, x_{d}\right), \quad i=1,2,3, \ldots, d,
$$

described in a $d$-dimensional state space and where $x \in \mathbb{R}^{d}$ are the variables. The vector of state variables will be designated by $\boldsymbol{x}$. Let $h: \mathbb{R}^{d}(\boldsymbol{x}) \rightarrow \mathbb{R}^{m}(\boldsymbol{s})$ be a measurement function such as it returns a single $m$-dimensional time series $\boldsymbol{s}(m \leqslant d)$. Starting with a scalar measured time series $(m=1)$ for the sake of simplicity (multivariate time series will be later considered in the subsequent part of this paper), it is possible to reconstruct a space $\mathbb{R}^{d}(\boldsymbol{X})$ from the time series $\left\{x_{i}(t)\right\}$ where $\boldsymbol{X} \in \mathbb{R}^{d}$ is the vector of the $d$-successive Lie derivative

$$
\boldsymbol{X}=\mid \begin{aligned}
& X_{1}=\mathcal{L}_{f}^{0} h(\boldsymbol{x}), \\
& X_{2}=\mathcal{L}_{f}^{1} h(\boldsymbol{x}), \\
& \vdots \\
& X_{d}=\mathcal{L}_{f}^{d-1} h(\boldsymbol{x}),
\end{aligned}
$$


and where $\mathcal{L}_{f}^{0} h(\boldsymbol{x})=h(\boldsymbol{x})$ is designated as the first Lie derivative (thus corresponding to the measured variable itself). Giving a vector field $\boldsymbol{f}(\boldsymbol{x})$ the Lie derivative of the $i$ th component of the vector field is defined as

$$
\mathcal{L}_{f} f_{i}(x)=\sum_{k=1}^{d} \frac{\partial f_{i}(x)}{\partial x_{k}} f_{k}(x)
$$

and the $n$-order derivative can be recursively calculated as

$$
\mathcal{L}_{f}^{n} f_{i}(\boldsymbol{x})=\mathcal{L}_{f}\left[\mathcal{L}_{f}^{n-1} f_{i}(\boldsymbol{x})\right]
$$

These Lie derivatives are assumed to span the reconstructed space $\mathbb{R}^{d}(\boldsymbol{X})$, sometimes named the differential embedding. The change of coordinates $\Phi_{s}: \mathbb{R}^{d}(\boldsymbol{x}) \rightarrow \mathbb{R}^{d}(\boldsymbol{X})$ is thus completely defined by the vector of Lie derivatives $\boldsymbol{X}$ whose Jacobian matrix corresponds to the so-called observability matrix for nonlinear systems [12].

System (1) is said to be fully observable if any two arbitrary state vectors $\boldsymbol{x}_{1}$ and $\boldsymbol{x}_{2}$ are distinguished with respect to the measurements $\boldsymbol{s}=h(\boldsymbol{x})$, that is, that $h\left(\boldsymbol{x}_{1}\right) \neq h\left(\boldsymbol{x}_{2}\right)$ if and only if $\boldsymbol{x}_{1} \neq \boldsymbol{x}_{2}$. This is equivalent to, say, that system (1) is observable when map $\Phi_{s}$ is invertible. Most of the time it is impossible to check the global invertibility but it is sufficient to prove that $\Phi_{s}\left(\boldsymbol{x}_{*}\right)$ is locally invertible at $\boldsymbol{x}_{*}$ by showing that the determinant of the Jacobian matrix $\mathcal{J}_{\Phi_{s}}$ is such as

$$
\operatorname{Det}\left[\mathcal{J}_{\Phi_{s}}\left(\boldsymbol{x}_{*}\right)\right] \neq 0 .
$$

If such a condition holds for every $\boldsymbol{x}_{*} \in \mathbb{R}^{d}(\boldsymbol{x})$, then $\Phi_{s}$ is said to be fully observable. The determinant $\operatorname{Det}\left[\mathcal{J}_{\Phi_{s}}\left(\boldsymbol{x}_{*}\right)\right]$ or equivalently $\operatorname{Det}\left[\mathcal{O}\left(\boldsymbol{x}_{*}\right)\right]$ since the observability matrix is by definition equal to $\mathcal{J}_{\Phi_{s}}$-is thus the relevant concept for determining whether a system is observable through some measurements or not.

\section{B. Observability and nonlinearity}

For the sake of simplicity, we consider in this paper only systems which cannot be decomposed into independent subsystems, that is, a system whose any row of its Jacobian matrix,

$$
\mathcal{J}_{f}\left(\boldsymbol{x}_{*}\right)=\left[\frac{\partial f}{\partial \boldsymbol{x}}\left(\boldsymbol{x}_{*}\right)\right],
$$

has at least one non-null off-diagonal element. This means that there is no variable whose time evolution is not dependent on the evolution of some others. Such a system is fully observable through the measurements $s$ if the change of coordinates $\Phi_{s}$ is such as $\operatorname{Det}\left[\mathcal{J}_{\Phi_{s}}\left(\boldsymbol{x}_{*}\right)\right] \neq 0\left[\forall \boldsymbol{x}_{*} \in \mathbb{R}^{d}(\boldsymbol{x})\right]$. Contrary to this, any point $\boldsymbol{x}_{*} \in \mathbb{R}^{d}(\boldsymbol{x})$ is nonobservable if the Jacobian matrix $\mathcal{J}_{\Phi_{s}}(\boldsymbol{x})$ is rank deficient, that is, if

$$
\operatorname{Det}\left[\mathcal{J}_{\Phi_{s}}\left(\boldsymbol{x}_{*}\right)\right]=0, \quad \text { for } \quad \boldsymbol{x}_{*} \in \mathbb{R}^{d}(\boldsymbol{x}) .
$$

In such a case, none of the states can be recovered from the measurement $\boldsymbol{x}_{*}$. This is very rarely the case for nondecomposable systems in the sense previously mentioned. Between these two extreme situations, there are some cases for which system (1) is nonobservable for a so-called singular observability manifold [13], that is, for a limited subset $\mathcal{M}_{\Phi_{s}}^{\text {obs }}$ of the state space $\mathbb{R}^{d}(\boldsymbol{x})$ for which $\operatorname{Det} \mathcal{J}_{\Phi_{s}}\left(\boldsymbol{x}_{*}\right)=0$.
These intermediary cases are the most often encountered as evidenced in $[11,12]$. They are associated with

$$
\operatorname{Det}\left[\mathcal{J}_{\Phi_{s}}\left(\boldsymbol{x}_{*}\right)\right]=g\left(\boldsymbol{x}_{*}\right)=0,
$$

where $g(\boldsymbol{x})$ is a nonconstant function depending on some variable(s) $x_{i}$, and $\boldsymbol{x}_{*} \in \mathcal{M}_{\Phi_{s}}^{\text {obs }}$. It thus appears that the lack of observability is strongly related by the presence of nonlinearities in the change of coordinates $\Phi_{s}[10,12]$.

Let us illustrate these statements using the Rössler system [14]

$$
\begin{aligned}
& \dot{x}=-y-z, \\
& \dot{y}=x+a y, \\
& \dot{z}=b+z(x-c),
\end{aligned}
$$

as an example. When variable $y$ is measured, the change of coordinates $\Phi_{y^{3}}$ between the original state space $\mathbb{R}^{3}(x, y, z)$ and the differential embedding $\mathbb{R}^{3}(X, Y, Z)$ reads

$$
\Phi_{y^{3}}=\mid \begin{aligned}
& X=y \\
& Y=\dot{y}=x+a y, \\
& Z=\ddot{y}=a x+\left(a^{2}-1\right) y-z .
\end{aligned}
$$

The exponent 3 in the subscript $y^{3}$ of $\Phi_{y^{3}}$ means that the first three Lie derivatives are considered for spanning the reconstructed space. We use such notation for any object which depends on not only the measured variables, but also on the way the Lie derivatives are chosen. The associated Jacobian matrix is

$$
\mathcal{J}_{\Phi_{y^{3}}}=\left[\begin{array}{ccc}
0 & 1 & 0 \\
1 & a & 0 \\
a & a^{2}-1 & -1
\end{array}\right]=\mathcal{O}_{y^{3}},
$$

which is also the observability matrix $\mathcal{O}_{y^{3}}$ of the Rössler system when variable $y$ is measured, and its first three Lie derivatives are considered. The corresponding determinant is thus

$$
\operatorname{Det}\left[\mathcal{J}_{\Phi_{y^{3}}}\right]=\operatorname{Det}\left[\mathcal{O}_{y^{3}}\right]=1,
$$

which never vanishes. The Rössler system is therefore fully observable from the measurements of variable $y$.

Let us now measure variable $x$ of the Rössler system. The change of coordinates becomes

$$
\Phi_{x^{3}}=\mid \begin{aligned}
& X=x \\
& Y=\dot{x}=-y-z, \\
& Z=\ddot{x}=-b-x-a y+c z-x z,
\end{aligned}
$$

and its Jacobian matrix

$$
\mathcal{J}_{\Phi_{x^{3}}}=\left[\begin{array}{ccc}
1 & 0 & 0 \\
0 & -1 & -1 \\
-1-z & -a & c-x
\end{array}\right]=\mathcal{O}_{x^{3}} .
$$

The associated determinant

$$
\operatorname{Det}\left[\mathcal{J}_{\Phi_{x^{3}}}\right]=\operatorname{Det}\left[\mathcal{O}_{x^{3}}\right]=x-(a+c)
$$

vanishes for the singular observability manifold $\mathcal{M}^{\text {obs }}$,

$$
\boldsymbol{x} \in \mathcal{M}_{x^{3}}^{\mathrm{obs}}=\left\{\boldsymbol{x} \in \mathbb{R}^{3} \mid x=a+c\right\} .
$$

The Rössler system is therefore observable from variable $x$ for any point $\boldsymbol{x}_{*} \in \mathbb{R}^{3}(x, y, z) \backslash \mathcal{M}_{x^{3}}^{\text {obs }}$, that is, for any point of the 
state space which does not belong to the singular observability manifold.

As a last example with the Rössler system, let us consider a measurement vector such as

$$
s=h(\boldsymbol{x})=\left[\begin{array}{lll}
1 & 0 & 0 \\
0 & 1 & 0 \\
0 & 0 & 0
\end{array}\right] \cdot \boldsymbol{x}^{\mathrm{T}},
$$

where $\boldsymbol{x}^{\mathrm{T}}$ is transpose vector of $\boldsymbol{x}$. The change of coordinates is such as

$$
\mathcal{J}_{\Phi_{x y^{2}}}=\mathcal{O}_{x y^{2}}=\left[\begin{array}{lll}
1 & 0 & 0 \\
0 & 1 & 0 \\
1 & a & 0
\end{array}\right],
$$

where the subscript $x y^{2}$ means that the first Lie derivative of $x$ and the first two ones of variable $y$ are considered; that is, $\Phi_{x y^{2}}=(x, y, \dot{y})^{\mathrm{T}}$. The singular observability manifold is indeed such as

$$
\mathcal{M}_{x y^{2}}^{\text {obs }}=\left\{\boldsymbol{x} \in \mathbb{R}^{3} \mid \operatorname{Det}\left[\mathcal{O}_{x y^{2}}\right]=0\right\}=\mathbb{R}^{3}(\boldsymbol{x}),
$$

and therefore, the system is never observable from these measurements. The tricky aspect of state space reconstruction from multivariate measurements is that it is not only important to correctly choose the set of measured variables but also to choose properly the variables whose successive Lie derivatives are added to complete the vector spanning the reconstructed space. Thus, if the third variable used for spanning the reconstructed space is the second Lie derivative of variable $x$, then the change of coordinates becomes

$$
\mathcal{O}_{x^{2} y}=\left[\begin{array}{rrr}
1 & 0 & 0 \\
0 & 1 & 0 \\
0 & -1 & -1
\end{array}\right]
$$

and its determinant is such as $\operatorname{Det}\left[\mathcal{O}_{x^{2} y}\right]=-1$, providing a full observability of the original state space of the Rössler system. As already pointed out [15], investigating the observability of a given system through multivariate measurements is not so straightforward because there are multiple choices for reconstructing a $d$-dimensional space from a $m$-dimensional set of measurements $(m<d)$. When the minimal set of variables is researched for reconstructing the full state of the system, the number of successive derivatives retained for each measured variables must be investigated too.

From these simple examples, it appears that the observability of a system through a variable is reduced by the nonconstant polynomial terms occurring in the computations of the determinant of the Jacobian matrix of the change of coordinates between the original state space and the reconstructed one. When considering rational system [16-18], it was remarked that rational terms in such a determinant were much more critical than nonconstant polynomial terms. These two types of terms have therefore a crucial role in the observability provided by a given variable.

\section{A new symbolic observability coefficient}

As we discussed above, there is a variety of cases between a full observability and a full lack of observability. To quantify this whole range of possibilities, observability coefficients, were introduced. The first definition of an observability coefficient was based on a numerical estimation of the eigenvalues of the observability matrix along a trajectory in the state space $[5,6]$. This coefficient had the disadvantage of not being normalized and, consequently, of not allowing comparison between these coefficients for different systems. Additionally, it required a combination of costly analytical and computational calculations which could only be applied to reasonably simple systems like three-dimensional rational systems or nine-dimensional quadratic systems, for instance. Based on a graphical approach [10], a symbolic coefficient $\breve{\eta}_{s}$ was then introduced in Ref. [11], thus avoiding analytical computations which can become difficult to handle when the dimension of the system considered is large (as we see in Sec. III G).

This symbolic coefficient $\breve{\eta}_{s}$ was not successful in detecting the full observability of one quadratic three-dimensional system. Therefore, we propose in this work a new symbolic observability coefficient $\eta_{s}$ which (i) no longer misses full observability, (ii) works correctly for rational systems, and (iii) is simpler to define (and to compute) since it is more closely related to analytical computations (but without their complexity). The main idea underlying the new coefficient $\eta_{s}$ is to count the number of each type of nonconstant terms occurring in the symbolic observability matrix $\mathcal{O}_{s}$, which is obtained from the symbolic Jacobian, a procedure described in detail in the following. In order to do that, there is no need to know exactly what are these terms, but only whether they are null, constant, polynomial, or rational.

The procedure is now explained using the Rössler system (9) from which only variable $x$ is measured. The case of higher-dimensional systems is straightforward and the case of multivariate measurements will be hereafter detailed. The steps for calculations of $\eta_{s}$ is as follows.

(1) Construct the symbolic Jacobian matrix $\tilde{\mathcal{J}}_{f}$ from the common Jacobian matrix $\mathcal{J}_{f}$ of the considered vector field $f$ by replacing each linear element $J_{i j}$ with 1 , each nonlinear polynomial element $J_{i j}$ with $\overline{1}$, and each rational element $J_{i j}$ with $\overline{\overline{1}}$ when the $j$ th variable is present in the denominator or by $\overline{1}$ otherwise.

Example. The Jacobian matrix

$$
\mathcal{J}_{f}=\left[\begin{array}{rrc}
0 & -1 & -1 \\
1 & 1 & 0 \\
z & 0 & x-c
\end{array}\right]
$$

of the Rössler system (9) is transformed in the symbolic Jacobian matrix

$$
\tilde{\mathcal{J}}_{f}=\left[\begin{array}{lll}
0 & 1 & 1 \\
1 & 1 & 0 \\
\overline{1} & 0 & \overline{1}
\end{array}\right] .
$$

(2) Construct the symbolic observability matrix $\tilde{\mathcal{O}}_{s}$ as follows. The first row defines the measurement vector $h$ for which we here assume that the single $j$ th component is nonzero (since we here consider only univariate measurements). The second row is directly obtained from the symbolic Jacobian matrix $\tilde{\mathcal{J}}_{f}$ by copying its $j$ th row (which, in fact, corresponds to the second Lie derivative of the measurement $s$, the first one being the measurement itself). The $k$ th row is obtained as follows: Transpose the $(k-1)$ th row of the symbolic 
observability matrix $\tilde{\mathcal{O}}_{s}$ just previously constructed, and then multiply each element $\tilde{\mathcal{J}}_{i j}$ of the symbolic Jacobian matrix $\tilde{\mathcal{J}}_{f}$ by the $i$ th component of the transposed $(k-1)$ th row. The multiplicative law between the symbols is defined by

$$
\begin{aligned}
& 0 \otimes \tilde{J}_{i j}=0, \\
& 1 \otimes \tilde{J}_{i j}=\tilde{J}_{i j}, \\
& \begin{array}{l}
\overline{1} \otimes \tilde{J}_{i j}=\mid \begin{array}{ll}
0 & \text { if } \tilde{J}_{i j}=0, \\
\overline{\overline{1}} & \text { if } \tilde{J}_{i j}=\overline{\overline{1}}, \\
\overline{1} & \text { otherwise, }
\end{array} \\
\overline{\overline{1}} \otimes \tilde{J}_{i j}=\mid \begin{array}{ll}
0 & \text { if } \tilde{J}_{i j}=0, \\
\overline{\overline{1}} & \text { otherwise. }
\end{array}
\end{array}
\end{aligned}
$$

The resulting symbolic Jacobian matrix $\tilde{\mathcal{J}}^{\prime}$ is thus transformed into a row by summing the elements of each column according to the addition law

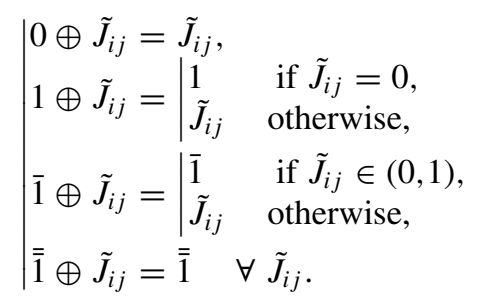

Example. Variable $x$ of the Rössler system is measured; the first row of $\tilde{\mathcal{O}}_{x^{3}}$ is thus

$$
\left[\begin{array}{lll}
1 & 0 & 0
\end{array}\right] \text {. }
$$

The second is therefore the first row of the symbolic Jacobian matrix $\tilde{\mathcal{J}}_{f}$ in Eq. (22); that is,

$$
\left[\begin{array}{lll}
0 & 1 & 1
\end{array}\right] \text {. }
$$

This row is now transposed and multiplied by the symbolic Jacobian matrix $\tilde{\mathcal{J}}_{f}$, leading to

$$
\left[\begin{array}{l}
0 \\
1 \\
1
\end{array}\right] \otimes\left[\begin{array}{lll}
0 & 1 & 1 \\
1 & 1 & 0 \\
\overline{1} & 0 & \overline{1}
\end{array}\right]=\left[\begin{array}{lll}
0 & 0 & 0 \\
1 & 1 & 0 \\
\overline{1} & 0 & \overline{1}
\end{array}\right] .
$$

Elements of the $j$ th column are summed to provide the $j$ th element of the third row of $\tilde{\mathcal{O}}_{x^{3}}$ as

$$
\left[\begin{array}{lll}
\overline{1} & 1 & \overline{1}
\end{array}\right] \text {. }
$$

The resulting symbolic observability matrix is thus

$$
\tilde{\mathcal{O}}_{x^{3}}=\left[\begin{array}{lll}
1 & 0 & 0 \\
0 & 1 & 1 \\
\overline{1} & 1 & \overline{1}
\end{array}\right] .
$$

(3) Compute the determinant of the symbolic observability matrix $\tilde{\mathcal{O}}_{s}$ but keep explicitly all terms actually contributing to the determinant (remove only the terms which are zero according to the multiplicative law $\otimes$ as previously introduced). Then count the number $N_{1}, N_{\overline{1}}$, and $N_{\overline{1}}$ of symbolic terms 1 , $\overline{1}$, and $\overline{\overline{1}}$, respectively, present in the determinant. The symbols presented in the rows coming from the measurement (first row in a univariate measurement [for example, Eq. (25)], or the $m-$ first rows in the case of multivariate [for example, Eqs. (31) and (32)], with $m<d$ the different variables measured, only contributing once to the counting. Also a special condition is required for rational systems that, if $N_{\overline{1}}=0$ and $N_{\overline{1}} \neq 0$, then $N_{\overline{1}}=N_{\overline{1}}$. The symbolic observability coefficients is then equal to

$$
\begin{aligned}
\eta_{s}= & \frac{N_{1}}{N_{1}+N_{\overline{1}}+N_{\overline{\overline{1}}}}+\frac{N_{\overline{1}}}{\left(N_{1}+N_{\overline{1}}+N_{\overline{1}}\right)^{2}} \\
& +\frac{N_{\overline{\overline{1}}}}{\left(N_{1}+N_{\overline{1}}+N_{\overline{1}}\right)^{3}} .
\end{aligned}
$$

Example. The determinant of the symbolic observability matrix $\tilde{\mathcal{O}}_{x^{3}}$ given in Eq. (29) is

$$
\operatorname{Det} \tilde{\mathcal{O}}_{x^{3}}=1 \otimes(1 \otimes \overline{1}-1 \otimes 1) .
$$

We have, therefore, $N_{1}=4, N_{\overline{1}}=1$, and $N_{\overline{1}}=0$, thus leading to

$$
\eta_{x^{3}}=\frac{4}{5}+\frac{1}{5^{2}}=0.84 .
$$

Similarly, we have

$$
\operatorname{Det} \tilde{\mathcal{O}}_{y^{3}}=1 \otimes(1 \otimes 1-1 \otimes 1),
$$

and, consequently, $N_{1}=5, N_{\overline{1}}=0$, and $N_{\overline{1}}=0$, leading to $\eta_{y^{3}}=1$. The third determinant is

$$
\operatorname{Det} \tilde{\mathcal{O}}_{z^{3}}=1 \otimes(\overline{1} \otimes \overline{1}-\overline{1} \otimes 0),
$$

leading to $N_{1}=1, N_{\overline{1}}=2$, and $N_{\overline{1}}=0$, which induce $\eta_{z^{3}}=$ $\frac{1}{3}+\frac{2}{3^{2}}=0.56$.

The case of multivariate measurements differs because there are $m$ rows with a single nonzero element in the observability matrix. The $(d-m)$ rows remaining to construct are obtained as detailed in step 2 where, for instance, the $(m+1)$ th row of the observability matrix corresponds to the $k$ th row of the symbolic matrix of Lie derivatives $\tilde{L}_{1}$ if variable $x_{k}$ is chosen, and the $(m+2)$ th row of $\tilde{\mathcal{O}}_{s}$ to the $k$ th row of $\tilde{L}^{2}$ when the next Lie derivative of variable $x_{k}$ is retained or by the $l$ th row of $\tilde{L}_{1}$ if the second Lie derivative of variable $x_{l}$ is preferred, and so on.

Example. Let us consider the case where variables $x$ and $y$ of the Rössler system are measured. The first two rows of the observability matrix $\tilde{\mathcal{O}}$ are thus

$$
\tilde{L}_{x}^{1}=\left[\begin{array}{lll}
1 & 0 & 0
\end{array}\right]
$$

and

$$
\tilde{L}_{y}^{1}=\left[\begin{array}{lll}
0 & 1 & 0
\end{array}\right] .
$$

Let us start by choosing the second Lie derivative of $x$ for spanning our reconstructed space. The third row is

$$
\tilde{L}_{x}^{2}=\left[\begin{array}{lll}
0 & 1 & 1
\end{array}\right],
$$

and the symbolic observability is

$$
\tilde{\mathcal{O}}_{x^{2} y}=\left[\begin{array}{lll}
1 & 0 & 0 \\
0 & 1 & 0 \\
0 & 1 & 1
\end{array}\right] .
$$

Its determinant is

$$
\operatorname{Det}\left[\tilde{O}_{x^{2} y}\right]=1 \otimes 1 \otimes 1 .
$$

In this determinant there are three constant elements contributing to the determinant; thus, $N_{1}=3, N_{\overline{1}}=0$, and $N_{\overline{1}}=0$, leading to $\eta_{x^{2} y}=1$. The Rössler system is thus fully observable when this embedding is used from the measurement 
of variables $x$ and $y$ and when the second Lie derivative of $x$ is chosen.

Let us now choose the second Lie derivative of variable $y$ and not the one of variable $x$. The third row is now

$$
\tilde{L}_{y}^{2}=\left[\begin{array}{lll}
1 & 1 & 0
\end{array}\right]
$$

and the observability matrix

$$
\tilde{\mathcal{O}}_{x y^{2}}=\left[\begin{array}{lll}
1 & 0 & 0 \\
0 & 1 & 0 \\
1 & 1 & 0
\end{array}\right]
$$

is rank deficient, so its determinant is directly equal to 0 . The symbolic observability coefficient is therefore also equal to zero since

$$
\operatorname{Det}\left[\tilde{O}_{x y^{2}}\right]=1 \otimes 1 \otimes 0=0,
$$

thus leading to $N_{1}=0, N_{\overline{1}}=0$, and $N_{\overline{1}}=0$, inducing $\eta_{x y^{2}}=0$.

\section{VALIDATION OF THE NEW SYMBOLIC OBSERVABILITY COEFFICIENT}

\section{A. Quadratic three-dimensional systems}

In order to validate our defined symbolic observability coefficient $\eta_{s}$, we started by comparing its values for some three-dimensional Sprott systems [19] which were investigated previously using the "old" symbolic coefficients $\breve{\eta}$ whose values can be seen in Table I in [11] and were reprinted in Table I, with the exception of a few values that were correctly recalculated (appearing in bold in Table I). In all cases but one the relative observability order-a ranking of observable variables from the one providing the best observability of the original state space to the one returning the worst observability — of the observability provided by the three variables was not modified in comparison with the new coefficient $\eta_{s}$; that is, the correct ranking of observable variables was detected by the new observability coefficient. Results are reported in Table I.

The single exception is for system "O" for which the observability ranking, obtained from the computation of $\breve{\eta}$, was $y \triangleright z \triangleright x$, where $\triangleright$ means "provides a better observability than," which differs from the ranking $y \triangleright x \triangleright z$ composed by our new coefficient $\eta$ (see Table I). Comparing the analytical determinant $\Delta_{x^{3}}$ and $\Delta_{z^{3}}$ of the observability matrices $\mathcal{O}_{x^{3}}$ and $\mathcal{O}_{z^{3}}$ (Table I), the former is a first-order polynomial and the latter a second-order polynomial: According to these properties, variable $z$ must provide an observability poorer than the one provided by variable $x$ since it is known that the higher the order of the polynomial, the less observable the state space is; see [6]. Therefore, the new observability coefficient $\eta_{s}$ defined in this work provides a better estimation of the observability than the one provided by the symbolic observability coefficient $\breve{\eta}$. Moreover, a mistake was discovered in the value obtained for $\breve{\eta}_{z^{3}}$ for system K, which was actually $\breve{\eta_{z^{3}}}=0.89$ (and not 1.00 , as wrongly reported in [11]). Consequently, the coefficient $\breve{\eta}_{z^{3}}$ missed the full observability provided by variable $z$ of system $\mathrm{K}$; the new coefficient returns $\eta_{z^{3}}=1.00$, which is a correct estimation of the full observability. We should thus consider that the new observability coefficient is validated for three-dimensional quadratic systems.

\section{B. A three-dimensional cubic system}

Among the various systems proposed by Rössler, let us investigate the cubic system [20],

$$
\begin{aligned}
& \dot{x}=-a x-y\left(1-x^{2}\right), \\
& \dot{y}=\mu(y+0.3 x-2 z), \\
& \dot{z}=\mu(x+2 y-0.5 z),
\end{aligned}
$$

which produces a double scroll attractor. The symbolic Jacobian matrix of this cubic system is

$$
\tilde{\mathcal{J}}_{f}=\left[\begin{array}{lll}
\overline{1} & \overline{1} & 0 \\
1 & 1 & 1 \\
1 & 1 & 1
\end{array}\right] .
$$

The new symbolic observability coefficients are $\eta_{x^{3}}=0.56$, $\eta_{y^{3}}=0.84$, and $\eta_{z^{3}}=0.68$, thus leading to the observability ranking $y \triangleright z \triangleright x$. This ranking slightly differs from the one found with the old symbolic observability coefficient [11]. Considering the analytic determinant of the observability matrices

$$
\begin{aligned}
& \Delta_{x^{3}}=-2 \mu\left(x^{2}-1\right)^{2}, \\
& \Delta_{y^{3}}=\mu^{2}(3.7 \mu+0.6 a-1.2 x y), \\
& \Delta_{z^{3}}=\mu^{2}\left(0.8 \mu+2 a-1+x^{2}-4 x y\right),
\end{aligned}
$$

one may remark that the first one is a fourth-order polynomial and that the last two determinants are second-order polynomials of a nearly equal complexity. The variable providing the poorest observability is the variable whose determinant $\Delta$ is a function of the highest order, as predicted by the new symbolic coefficient $\eta_{s}$. It is not surprising that our new symbolic observability coefficient estimated the observability provided by variables $y$ and $z$ to be large. This was confirmed by the global models of equal quality which were found from these two variables, as discussed in [11].

\section{A three-dimensional quadratic system with symmetry}

The Lorenz system [21]

$$
\begin{aligned}
& \dot{x}=\sigma(y-x), \\
& \dot{y}=R x-y-x z, \\
& \dot{z}=-b z+x y,
\end{aligned}
$$

is known for presenting a rotation symmetry around the $z$ axis under which the Lorenz attractor is globally left invariant. This symmetry property can be expressed using the $3 \times 3$ matrix

$$
\Gamma=\left[\begin{array}{rrr}
-1 & 0 & 0 \\
0 & -1 & 0 \\
0 & 0 & 1
\end{array}\right],
$$

which is characteristic of that symmetry. The Lorenz system $\boldsymbol{f}(\boldsymbol{x})$ thus satisfies

$$
\Gamma \cdot \dot{x}=f(\Gamma \cdot \boldsymbol{x}) .
$$

The (Lorenz) system $\boldsymbol{f}$ is said to be equivariant under the rotation symmetry so defined by the $\Gamma$ matrix [22]. 
TABLE I. Sets of equations investigated with the determinant $\Delta_{i}{ }^{3}$ of the Jacobian matrix of the change of coordinates $\Phi_{i}{ }^{3}$ between the original state space and the space reconstructed from the $i$ th variable using its successive Lie derivatives. The system is fully observable when $\Phi_{i^{3}}$ defines a global diffeomorphism, that is, when $\eta_{i^{3}}=1$. Values of $\breve{\eta}$ in bold fonts are those which were correctly recalculated, compared to those values reported in [11].

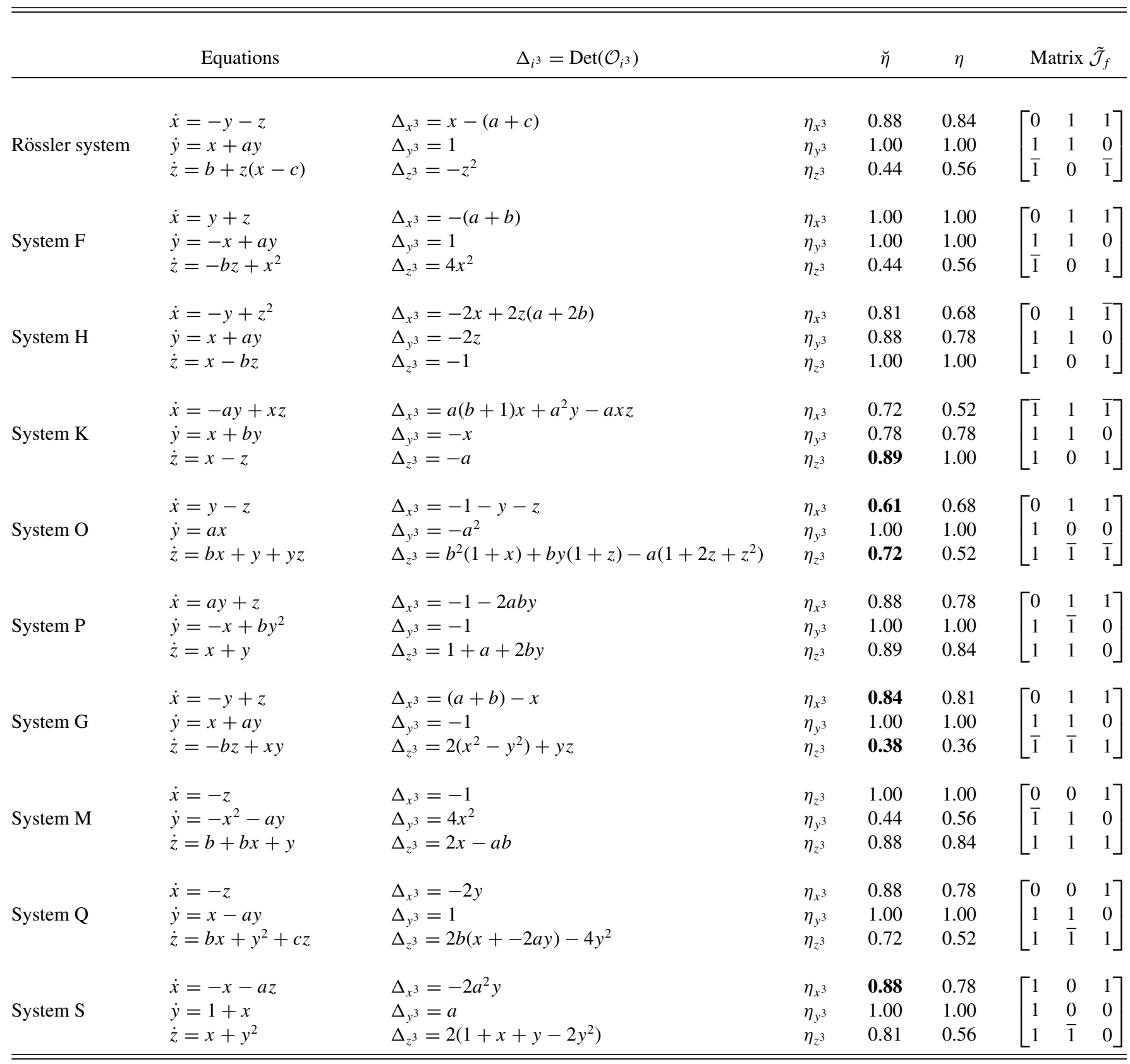

The symbolic Jacobian matrix of the Lorenz system is

$$
\tilde{\mathcal{J}}_{f}=\left[\begin{array}{lll}
1 & 1 & 0 \\
\overline{1} & 1 & \overline{1} \\
\overline{1} & \overline{1} & 1
\end{array}\right] .
$$

The three symbolic observability coefficients

$$
\eta_{x^{3}}=0.75>\eta_{y^{3}}=0.16=\eta_{z^{3}}=0.16
$$

are in agreement with the three analytical determinants of the analytical Jacobian matrix. Indeed, these three determinants are

$$
\begin{aligned}
& \Delta_{x^{3}}=-\sigma^{2} x \\
& \Delta_{y^{3}}=R(\sigma y-b x)-\sigma y z+2 x^{2} y, \\
& \Delta_{z^{3}}=2\left[(z-R) x^{2}+\sigma y^{2}\right] .
\end{aligned}
$$

The first one is a first-order polynomial; the last two ones are both third-order polynomials. Variable $x$ therefore provides the best observability of the original state space [6]. The two other variables provide a lower quality for reconstructing the original state space. 
The symmetry also plays an important role in reconstructing the state space. For instance, as already pointed out in [23], the $x$-induced differential embedding is associated with an attractor left invariant under an inversion symmetry and no longer with a rotation symmetry. Contrary to this, when variable $z$ is measured, the resulting differential embedding produces an attractor without any residual symmetry: It is the result from a $2 \mapsto 1$ mapping which mods out the rotation symmetry [22]. The singular observability manifold

$$
\mathcal{M}_{z^{3}}^{\text {obs }}=\left\{(x, y, z) \in \mathbb{R}^{3} \mid z=R-\sigma\left(\frac{y}{x}\right)^{2}\right\}
$$

is quite complex (see Fig. 6(c) in [13]). The key point is that, from the theoretical point of view, any points $( \pm x, \pm y, z) \in$ $\mathbb{R}^{3} \backslash \mathcal{M}_{z^{3}}^{\text {obs }}$ from the original state space and which does not belong to the singular observability manifold is mapped into a single point in the $z$-induced differential embedding $\mathbb{R}^{3}\left(\boldsymbol{X}_{z^{3}}\right)$ : This variable therefore never distinguishes symmetry-related points. Thus variable $z$ does not provide any observability of the original state space stricto sensu. This means that, rigorously speaking, the full state space is not observable (or strongly indistinguishable in the sense of Hermann and Kerner [4]) and, consequently, the symbolic observability coefficient $\eta_{z^{3}}$ should be zero. This clearly shows that the computation of the observability only considers the singular observability manifold and not the possible symmetries the studied system may have.

In order to reconstruct a space with the rotation symmetry the Lorenz system has, it is necessary to use at least two variables, one which is mapped into its opposite and one which is left invariant under the rotation. Two possible sets of measurements are thus possible: $(x, z)$ and $(y, z)$. To restore the rotation symmetry, it is necessary to take the second Lie derivative of the variable which is mapped into its opposite under the rotation. We have thus to consider the observability matrices $\tilde{\mathcal{O}}_{x^{2} z}$ and $\tilde{\mathcal{O}}_{y^{2} z}$. We obtained $\eta_{x^{2} z}=1.00$ and $\eta_{y^{2} z}=$ 0.78 , which are in agreement with the determinants

$$
\Delta_{x^{2} z}=\sigma
$$

which never vanishes, and

$$
\Delta_{y^{2} z}=z-R
$$

which is a first-order polynomial, respectively. Note that if we would have used the second Lie derivatives of $z$ rather than of $x$, we would have obtained $\eta_{x z^{2}}=0.78$, that is, the same value as $\eta_{y^{2} z}$, thus ignoring the fact that the reconstructed space would present a reflection symmetry (a symmetry that a given attractor can never have) and not a rotation. It is therefore relevant to note that symmetry properties are badly considered by an observability analysis and that the results should be carefully interpreted for systems presenting equivariant properties.

\section{Three-dimensional rational systems}

Let us start with the example of a three species food chain described by [24]

$$
\begin{aligned}
& \dot{x}=x(1-x)-\frac{x y}{x+a}, \\
& \dot{y}=-b y+\frac{c x y}{x+d}-\frac{y z}{y+e}, \\
& \dot{z}=f z^{2}-\frac{g z^{2}}{y+h}
\end{aligned}
$$

where $x$ is the normalized density of preys (rodents) at the bottom of the food chain, $y$ the density of a specialist predator (snakes), and $z$ the density of the generalist predator (peacocks). The symbolic Jacobian matrix of this system reads

$$
\tilde{\mathcal{J}}_{f}=\left[\begin{array}{lll}
\overline{\overline{1}} & \overline{1} & 0 \\
\overline{\overline{1}} & \overline{\overline{1}} & \overline{1} \\
0 & \overline{\overline{1}} & \overline{1}
\end{array}\right],
$$

and the symbolic observability coefficients are

(i) $\eta_{x^{3}}=0.56$, with $N_{1}=1, N_{\overline{1}}=2$, and $N_{\overline{1}}=0$;

(ii) $\eta_{y^{3}}=0.26$, with $N_{1}=1, N_{\overline{1}}=1$, and $N_{\overline{1}}=3$;

(iii) $\eta_{z^{3}}=0.30$, with $N_{1}=1, N_{\overline{1}}=2$, and $N_{\overline{1}}=2$.

We thus have the observability ranking $x \triangleright z \triangleright y$, which is in agreement with the ranking previously obtained [16]. The corresponding analytical determinants are

$$
\begin{aligned}
\Delta_{x^{3}}= & -\frac{x^{2} y}{(x+a)^{2}(y+e)} \\
\Delta_{y^{3}} \text { too long with higher polynomical orders } & \\
& \text { than } \Delta_{x^{3}} \text { and } \Delta_{z^{3}} \\
\Delta_{z^{3}}= & -\frac{c d g^{2} y z^{4}}{(x+d)^{2}(y+h)^{4}} .
\end{aligned}
$$

The observability ranking is mostly in agreement with the increasing complexity of the determinants of the analytical observability matrices. Nevertheless, the number of terms was too large to attempt a simplification of the expression of $\Delta_{y^{3}}$ and to be able to compare it with $\Delta_{z^{3}}$. On the other hand, the ranking we found is confirmed by our ability to get a global model from the different variables as discussed in [16] since a global model was easily obtained from variables $x$ and $y$, but with a greater difficulty and less accuracy from variable $z$.

A second example of a three-dimensional rational system consists of a simple cancer model [25], here rewritten as [17]

$$
\begin{aligned}
& \dot{x}=\rho_{1} x(1-x)-\alpha_{13} x z, \\
& \dot{y}=\frac{\rho_{2} y z}{1+z}-\alpha_{23} y z-\delta_{2} y, \\
& \dot{z}=z(1-z)-x z-\alpha_{32} y z,
\end{aligned}
$$

where $x$ is the normalized population of host cells, $y$ the population of immune cells, and $z$ the population of tumor cells. The symbolic Jacobian matrix reads

$$
\tilde{\mathcal{J}}_{f}=\left[\begin{array}{lll}
\overline{1} & 0 & \overline{1} \\
0 & \overline{1} & \overline{1} \\
\overline{1} & \overline{1} & \overline{1}
\end{array}\right] .
$$

The symbolic observability coefficients are $\eta_{x^{3}}=0.56, \eta_{y^{3}}=$ 0.30 , and $\eta_{z^{3}}=0.36$, thus leading to the ranking $x \triangleright z \triangleright y$, as obtained in [17] using numerical estimation of the observability coefficients. Once again, this is also in agreement with the complexity of the analytical determinants since

$$
\begin{aligned}
\Delta_{x^{3}}= & -\alpha_{13}^{2} \alpha_{32} x^{2} z ; \\
\Delta_{y^{3}}= & y^{2} z\left[\frac{\rho_{2}}{(1+z)^{4}}-\alpha_{23}\right] \\
& \times\left[\alpha_{23}-\rho_{2}+\alpha_{23} z(2+z)\right] ;
\end{aligned}
$$




$$
\begin{aligned}
\Delta_{z^{3}}= & -\alpha_{23} z^{2}\left[\left(\alpha_{23}-\alpha_{13}\right) z\right. \\
& \left.+\rho_{1}(1-2 x)+\frac{z\left(\alpha_{13} \alpha_{32} x^{2}-\rho_{2}\right)}{1+z}\right] .
\end{aligned}
$$

Note that $\Delta_{z^{3}}$ is actually simpler since it has a first-order denominator and not a fourth-order one as $\Delta_{y^{3}}$. With these two examples, we can show that our symbolic observability coefficients are well estimating the observability for rational systems, a feature which was not observed with the "old" symbolic observability coefficient developed in [11].

\section{E. A pathological rational system}

Let us consider the three-dimensional rational systems describing the dynamics of a biochemical model presented in [26]. A substrate is transformed by enzyme $E_{1}$ into product $P_{1}$, which serves as substrate for a second enzyme $E_{2}$ that transforms $\mathrm{P}_{1}$ into $\mathrm{P}_{2}$. The system thus reads

$$
\begin{aligned}
& \dot{x}=V-\sigma_{1} \phi_{1}(x, y), \\
& \dot{y}=q_{1} \sigma_{1} \phi_{1}(x, y)-\sigma_{2} \phi_{2}(y, z), \\
& \dot{z}=q_{2} \sigma_{2} \phi_{2}(y, z)-K_{s} z,
\end{aligned}
$$

where $x, y$, and $z$ are normalized using the concentrations of substrate $\mathrm{S}$ and of the reaction products $\mathrm{P}_{1}$ and $\mathrm{P}_{2}$, respectively. $\sigma_{1}$ and $\sigma_{2}$ are the normalized maximum rates of the enzyme $\mathrm{E}_{1}$ and $\mathrm{E}_{2}, q_{1}$ and $q_{2}$ quantify the ratios of the dissociation constants, $V$ denotes the substrate injection rate, and $K_{s}$ is the apparent first-order rate constant for the removal of the final product. The rate functions $\phi_{1}$ and $\phi_{2}$ of the allosteric enzymes $\mathrm{E}_{1}$ and $\mathrm{E}_{2}$ are

$$
\begin{aligned}
\phi_{1}(x, y) & =\frac{x(1+x)(1+y)^{2}}{L_{1}+(1+x)^{2}(1+y)^{2}}, \\
\phi_{2}(y, z) & =\frac{y(1+z)^{2}}{L_{2}+(1+z)^{2}} .
\end{aligned}
$$

Parameter values for producing chaotic solutions were $\sigma_{1}=$ $10 \mathrm{~s}^{-1}, q_{1}=50, L_{1}=5 \times 10^{8}, \sigma_{2}=10 \mathrm{~s}^{-1}, q_{2}=0.02, K_{s}=$ 2, and $L_{2}=100$ [26]. The corresponding symbolic Jacobian matrix reads

$$
\tilde{\mathcal{J}}_{f}=\left[\begin{array}{lll}
\overline{\overline{1}} & \overline{\overline{1}} & 0 \\
\overline{\overline{1}} & \overline{\overline{1}} & \overline{\overline{1}} \\
0 & \overline{1} & \overline{\overline{1}}
\end{array}\right] .
$$

The symbolic observability coefficients are $\eta_{x^{3}}=0.30, \eta_{y^{3}}=$ 0.17 , and $\eta_{z^{3}}=0.48$, leading to the observability ranking $z \triangleright x \triangleright y$, which contradicts the ranking provided by the numerical estimations of the observability coefficients and the global models we obtained from the three variables [18] (i.e., $x \triangleright z \triangleright y)$.

In order to understand this disagreement, it is helpful to remark the extremely large value of $L_{1}$, which leads a possible approximation of the rate function by

$$
\phi_{1}^{\prime}(x, y) \approx \frac{x(1+x)(1+y)^{2}}{L_{1}} .
$$

The value of parameter $L_{2}$ is large enough to only allow fluctuations smaller than $2 \%$ in the denominator of $\phi_{2}(y, z)$, which can also be approximated by

$$
\phi_{2}^{\prime}(y, z) \approx \frac{y(1+z)^{2}}{L_{2}} .
$$

Using this approximated function $\phi_{1}^{\prime}$, the symbolic Jacobian matrix becomes

$$
\tilde{\mathcal{J}}_{f}^{\prime}=\left[\begin{array}{lll}
\overline{1} & \overline{1} & 0 \\
\overline{1} & \overline{1} & \overline{1} \\
0 & \overline{1} & \overline{1}
\end{array}\right],
$$

since both nonlinear terms in the denominators of the rate function in Eq. (55) do not significantly contribute to the rate functions and for this reason we neglect them. Resolving its determinant, we obtain that the symbolic observability coefficients are $\eta_{x^{3}}=0.56, \eta_{y^{3}}=0.36$, and $\eta_{z^{3}}=0.56$. The approximated observability ranking is thus $x \bumpeq z \triangleright y$, where $\bumpeq$ would mean that variables $x$ and $z$ would provide the same observability of the original state space in such an approximation. This suggests that the estimation obtained in the rigorous case is also biased and that the correct answer should be, after all, the numerical observability ranking $x \triangleright z \triangleright y$ obtained in [18]. This is the single discrepancy between the observability ranking provided by our new symbolic observability coefficients with more rigorous results as analytical determinants of the observability matrix, but note that the present failure was obtained in a case where the nonlinearities are rather pathological. This example suggests to use approximated equations for the rate function when terms are obviously negligible compared to others.

\section{F. A four-dimensional quadratic system}

Let us now consider a four-dimensional quadratic system producing a hyperchaotic attractor [27]. It reads

$$
\begin{aligned}
\dot{x} & =-y-z, \\
\dot{y} & =x+a y+w, \\
\dot{z} & =b+x z, \\
\dot{w} & =-c z+d w .
\end{aligned}
$$

Its symbolic Jacobian matrix reads

$$
\tilde{\mathcal{J}}_{f}=\left[\begin{array}{llll}
0 & 1 & 1 & 0 \\
1 & 1 & 0 & 1 \\
\overline{1} & 0 & \overline{1} & 0 \\
0 & 0 & 1 & 1
\end{array}\right],
$$

and the corresponding symbolic observability coefficients are

(i) $\eta_{x^{4}}=0.79$, with $N_{1}=10, N_{\overline{1}}=3$, and $N_{\overline{1}}=0$;

(ii) $\eta_{y^{4}}=0.79$, with $N_{1}=10, N_{\overline{1}}=3$, and $N_{\overline{1}}=0$;

(iii) $\eta_{z^{4}}=0.44$, with $N_{1}=1, N_{\overline{1}}=3$, and $N_{\overline{1}}=0$;

(iv) $\eta_{w^{4}}=0.63$, with $N_{1}=2, N_{\overline{1}}=2$, and $N_{\overline{1}}=0$.

The observability ranking is thus $y \bumpeq x \triangleright w \triangleright z$, where $\bumpeq$ means "provides the same observability as." This ranking is slightly different from the previously obtained ranking [10,11] since the first two symbolic observability coefficients are equal. According to the determinants

(i) $\Delta_{x^{4}}=a d-c-x(a+d)-y-z+x^{2} \quad$ (second order),

(ii) $\Delta_{y^{4}}=d^{2}-x d(1+c)+z\left(1+2 c+c^{2}\right)$ (first order), 
(iii) $\Delta_{z^{4}}=z^{3}$ (third order),

(iv) $\Delta_{w^{4}}=c^{3} z^{2}$ (second order),

variable $y$ should provide a slightly better observability since the corresponding determinant is of first order and not of second order, as obtained for variable $x$. Note that replacing the fourth-order Lie derivative of $x$ with a measurement of variable $y$ or $z$ leads to a full observability since $\eta_{x^{3} y}=\eta_{x^{3} z}=1$ but not with variable $w$ since $\Delta_{x^{3} w}=x-a$ and for which $\eta_{x^{3} m}=0.86$. There are a variety of sets of measured variables which provide a full observability, for example, $\Phi_{y^{3} x}, \Phi_{y^{3} z}$, $\Phi_{y^{3} w}, \Phi_{x^{2} y^{2}}, \Phi_{x^{2} w^{2}}$, and $\Phi_{y^{2} w^{2}}$.

\section{G. A 5D rational system}

A model for the circadian oscillation in the Drosophila period protein was proposed by Goldbeter [28]. This is the five-dimensional rational model

$$
\begin{aligned}
& \dot{x}_{1}=\frac{v_{s} K_{I}^{4}}{K_{I}^{4}+x_{5}^{4}}-\frac{v_{m} x_{1}}{K_{m}+x_{1}}, \\
& \dot{x}_{2}=k_{s} x_{1}-\frac{V_{1} x_{2}}{K_{1}+x_{2}}+\frac{V_{2} x_{3}}{K_{2}+x_{3}}, \\
& \dot{x}_{3}=\frac{V_{1} x_{2}}{K_{1}+x_{2}}+\frac{V_{4} x_{4}}{K_{4}+x_{4}}-x_{3}\left(\frac{V_{2}}{K_{2}+x_{3}}+\frac{V_{3}}{K_{3}+x_{3}}\right), \\
& \dot{x}_{4}=\frac{V_{3} x_{3}}{K_{3}+x_{3}}-x_{4}\left(\frac{V_{4}}{K_{4}+x_{4}}+k_{1}+\frac{v_{d}}{K_{d}+x_{4}}\right), \\
& \dot{x}_{5}=k_{1} x_{4}-k_{2} x_{5},
\end{aligned}
$$

which produces a limit cycle for the parameter values reported in [28]. This system is interesting in the sense that its complexity presents a big challenge for analytical computation. The corresponding symbolic Jacobian matrix reads

$$
\tilde{\mathcal{J}}_{f}=\left[\begin{array}{lllll}
\overline{\overline{1}} & 0 & 0 & 0 & \overline{\overline{1}} \\
1 & \overline{\overline{1}} & \overline{\overline{1}} & 0 & 0 \\
0 & \overline{\overline{1}} & \overline{\overline{1}} & \overline{\overline{1}} & 0 \\
0 & 0 & \overline{\overline{1}} & \overline{\overline{1}} & 0 \\
0 & 0 & 0 & 1 & 1
\end{array}\right],
$$

and the corresponding symbolic observability coefficients are

(i) $\eta_{x_{1}^{5}}=0.17$, with $N_{1}=1, N_{\overline{1}}=4$, and $N_{\overline{\overline{1}}}=4$;

(ii) $\eta_{x_{2}^{5}}=0.08$, with $N_{1}=7, N_{\overline{1}}=42$, and $N_{\overline{1}}=42$;

(iii) $\eta_{x_{3}^{5}}=0.02$, with $N_{1}=1, N_{\overline{1}}=32$, and $N_{\overline{1}}=32$;

(iv) $\eta_{x_{4}^{5}}=0.17$, with $N_{1}=1, N_{\overline{1}}=4$, and $N_{\overline{1}}=4$.

(v) $\eta_{x_{5}^{5}}=0.30$, with $N_{1}=2, N_{\overline{1}}=3$, and $N_{\overline{1}}=3$,

thus leading to the observability ranking

$$
x_{5} \triangleright x_{1} \bumpeq x_{4} \triangleright x_{2} \triangleright x_{3} .
$$

This is in a rather good agreement to the analytical determinants

$$
\Delta_{x_{1}^{5}}=\frac{256 v_{s}^{4} K_{I}^{16} k_{1}^{3} V_{3}^{2} K_{3}^{2} V_{1} K_{1}}{\left(K_{I}^{4}+x_{5}^{4}\right)^{8}\left(K_{3}+x_{3}\right)^{4}\left(K_{1}+x_{2}\right)^{2}} x_{5}^{12}
$$

$\Delta_{x_{2}^{5}}$ and $\Delta_{x_{3}^{5}}$ where the complexity exceeds the order of the other determinants;

$$
\begin{aligned}
& \Delta_{x_{4}^{5}}=\frac{4 V_{3}^{4} K_{3}^{4} V_{1}^{3} K_{1}^{3} k_{s}^{2} v_{s} K_{I}^{4}}{\left(K_{3}+x_{3}\right)^{8}\left(K_{1}+x_{2}\right)^{6}\left(K_{I}^{4}+x_{5}^{4}\right)^{2}} x_{5}^{3} ; \\
& \Delta_{x_{5}^{5}}=-\frac{k_{1}^{4} V_{3}^{3} K_{3}^{3} V_{1}^{2} K_{1}^{2} k_{s}}{\left(K_{3}+x_{3}\right)^{6}\left(K_{1}+x_{2}\right)^{4}} .
\end{aligned}
$$

The symbolic observability matrix $\tilde{\mathcal{O}}_{x_{i}}^{5}$ can be easily obtained despite of the complexity of the system. By investigating the symbolic observability matrices, we found that the best measurement (when there are some nonmeasured variables) was the set $\left(x_{1}, x_{2}, x_{3}\right)$ and using the embedding provided by $\Phi_{x_{2}^{2} x_{3} x_{5}^{2}}$ : In such a case, there is a full observability since $\eta_{x_{2}^{2} x_{3} x_{5}^{2}}=1$ as confirmed by $\Delta_{x_{2}^{2} x_{3} x_{5}^{2}}=k_{s} k_{1}$. A local observability is obtained with $\Phi_{x_{2}^{2} x_{3}^{2} x_{5}}$, as confirmed by $\eta_{x_{2}^{2} x_{3}^{2} x_{5}}=0.70$ and the determinant

$$
\Delta_{x_{2}^{2} x_{3}^{2} x_{5}}=-\frac{k_{s} V_{4} K_{4}}{\left(K_{4}+x_{4}\right)^{2}} .
$$

A full lack of observability is obtained with $\Phi_{x_{2} x_{3}^{2} x_{5}^{2}}$ since $\tilde{\mathcal{O}}_{x_{2} x_{3}^{2} x_{5}^{2}}$ is rank deficient. It is here therefore demonstrated that our symbolic observability coefficients are useful for assessing the observability of quite high-dimensional rational systems.

\section{CONCLUSION}

Observability coefficients make it possible to assess the observability provided by some measurements to reconstruct a space in which distinct states in the original state space are distinguished. Assessing the observability of quite complicated systems due to their large dimensionality and/or their rational nature cannot be performed using analytical computations. In this work we proposed a new symbolic observability coefficient which not only corrects the previous one but also makes it possible to investigate rational systems. We construct a symbolic observability matrix whose determinant is symbolically computed, allowing the counting of the number of elements of the symbolic Jacobian matrix contributing to it. We checked the validity of our results with the analytical determinants or with some numerical estimations previously reported. It remains that, as with any observability analysis, the results must be carefully interpreted when systems with symmetry properties are considered since any symmetry, a global property, is poorly considered by local analysis.

\section{ACKNOWLEDGMENTS}

E.B.M. and M.S.B. acknowledge the Engineering and Physical Sciences Research Council (EPSRC), Grant No. EP/I032608/1. This work was done during a stay of E.B.M. at CORIA (Rouen) and a stay of C.L. at ICSMB (Aberdeen). 
[1] F. Takens, Detecting strange attractors in turbulence, Lect. Notes Math. 898, 366 (1981).

[2] D. Aeyels, Generic observability of differentiable systems, SIAM J. Control Optim. 19, 595 (1981).

[3] J. F. Gibson, J. D. Farmer, M. Casdagli, and S. Eubank, An analytic approach to practical state space reconstruction, Physica D (Amsterdam, Neth.) 57, 1 (1992).

[4] R. Hermann and A. Krener, Nonlinear controllability and observability, IEEE Trans. Autom. Control 22, 728 (1977).

[5] C. Letellier, J. Maquet, L. Le Sceller, G. Gouesbet, and L. A. Aguirre, On the non-equivalence of observables in phase space reconstructions from recorded time series, J. Phys. A 31, 7913 (1998).

[6] C. Letellier and L. A. Aguirre, Investigating nonlinear dynamics from time series: The influence of symmetries and the choice of observables, Chaos 12, 549 (2002).

[7] L. A. Aguirre and C. Letellier, Investigating observability properties from data in nonlinear dynamics, Phys. Rev. E 83, 066209 (2011).

[8] L. A. Aguirre, Controllability and observability of linear systems: some noninvariant aspects, IEEE Trans. Educ. 38, 33 (1995).

[9] U. Parlitz, J. Schumann-Bischoff, and S. Luther, Local observability of state variables and parameters in nonlinear modeling quantified by delay reconstruction, Chaos 24, 024411 (2014).

[10] C. Letellier and L. A. Aguirre, A graphical interpretation of observability in terms of feedback circuits, Phys. Rev. E 72, 056202 (2005).

[11] C. Letellier and L. A. Aguirre, Symbolic observability coefficients for univariate and multivariate analysis, Phys. Rev. E 79, 066210 (2009).

[12] C. Letellier, L. A. Aguirre, and J. Maquet, Relation between observability and differential embeddings for nonlinear dynamics, Phys. Rev. E 71, 066213 (2005).

[13] M. Frunzete, J.-P. Barbot, and C. Letellier, Influence of the singular manifold of nonobservable states in reconstructing chaotic attractors, Phys. Rev. E 86, 026205 (2012).

[14] O. E. Rössler, An equation for continuous Chaos, Phys. Lett. A 57, 397 (1976).
[15] L. A. Aguirre and C. Letellier, Observability of multivariate differential embeddings, J. Phys. A 38, 6311 (2005).

[16] C. Letellier, L. A. Aguirre, J. Maquet, and M. A. Aziz-Alaoui, Should all the species of a food chain be counted to investigate the global dynamics? Chaos Solitons Fractals 13, 1099 (2002).

[17] C. Letellier, F. Denis, and L. A. Aguirre, What can be learned from a chaotic cancer model? J. Theor. Biol. 322, 7 (2013).

[18] C. Letellier, L. A. Aguirre, and J. Maquet, How the choice of the observable may influence the analysis of non linear dynamical systems, Commun. Nonlinear Sci. Numer. Simul. 11, 555 (2006).

[19] J. C. Sprott, Some simple chaotic flows, Phys. Rev. E 50, R647 (1994).

[20] O. E. Rössler, Chemical turbulence: a synopsis, in Synergetics, edited by H. Haken, Proceedings of the International Workshop on Synergetics (Springer-Verlag, New York, 1977), pp. 174183.

[21] E. N. Lorenz, Deterministic nonperiodic flow, J. Atmos. Sci. 20, 130 (1963).

[22] C. Letellier and R. Gilmore, Covering dynamical systems: Twofold covers, Phys. Rev. E 63, 016206 (2001).

[23] C. Letellier and G. Gouesbet, Topological characterization of reconstructed attractors modding out symmetries, J. Phys. II (France) 6, 1615 (1996).

[24] R. K. Upadhyay, S. R. K. Jyengar, and V. Rai, Chaos: An ecological reality? Int. J. Bifurcation Chaos Appl. Sci. Eng. 8, 1325 (1998).

[25] L. G. de Pillis and A. Radunskaya, The dynamics of an optimally controlled tumor model: A case study, Math. Comput. Model. 37, 1221 (2003).

[26] O. Decroly and A. Goldbeter, Birhythmicity, chaos and other patterns of temporal self-organization in a multiply regulated biochemical system, Proc. Natl. Acad. Sci. USA 79, 6917 (1982).

[27] O. E. Rössler, An equation for hyperchaos, Phys. Lett. A 71, 155 (1979).

[28] A. Goldbeter, A model for circadian oscillations in the Drosophila period protein, Proc. R. Soc. London, Ser. B 261, 319 (1995). 